\title{
Análisis nutricional de tres alimentos balanceados para cachorros, fabricados y comercializados en Ecuador
}

\author{
Nutritional analysis of three balanced foods for puppies, manufactured \\ and marketed in Ecuador
}

Renán Mena-Pérez ${ }^{1,2,3}$, Angélica Madero-Guerrero ${ }^{1,}$ María Villanueva-Espinoza $^{2}$

\section{Resumen}

\begin{abstract}
El objetivo del estudio fue determinar la calidad nutricional de tres alimentos comerciales «Premium» para cachorros fabricados y comercializados en el Ecuador, así como determinar la sensibilidad de la técnica de espectroscopía por infrarrojo cercano (NIRS) para la valoración de los macronutrientes de estos alimentos. Se analizaron las características nutricionales de tres marcas de alimento, tomándose muestras de cuatro lotes por marca. Se determinaron los macronutrientes (proteína, grasa, fibra, almidón, cenizas y humedad) mediante la técnica NIRS, prueba que fue duplicada en dos laboratorios con diferentes equipos. Además, se determinó el perfil de aminoácidos mediante cromatografía líquida (HPLC). En el análisis proximal, los tres alimentos cumplen con los requerimientos de macronutrientes mínimos para garantizar el desarrollo y la salud de los cachorros, aunque uno de ellos presentó una mejor composición nutricional. La técnica de NIRS como prueba rápida es estable para analizar los componentes de los alimentos secos para mascotas; sin embargo, presentó variabilidad al medir principalmente la concentración de grasa $(\mathrm{p}=0.029)$ en los tres alimentos, aunque también ocurrió en uno de los
\end{abstract}

${ }^{1}$ Facultad de Medicina Veterinaria y Zootecnia, Universidad Central del Ecuador, Quito, Ecuador

${ }^{2}$ Escuela de Postgrado, Universidad Nacional Agraria La Molina, Lima, Perú

${ }^{3}$ E-mail: rpmena@uce.edu.ec

Recibido:11 de marzo de 2021

Aceptado para publicación: 18 de septiembre de 2020

Publicado: 27 de octubre de 2021

CLos autores. Este artículo es publicado por la Rev Inv Vet Perú de la Facultad de Medicina Veterinaria, Universidad Nacional Mayor de San Marcos. Este es un artículo de acceso abierto, distribuido bajo los términos de la licencia Creative Commons Atribución 4.0 Internacional (CC BY 4.0) [https:// creativecommons.org/licenses/by/4.0/deed.es] que permite el uso, distribución y reproducción en cualquier medio, siempre que la obra original sea debidamente citada de su fuente original 
alimentos para los contenidos de fibra y cenizas. La concentración de aminoácidos no esenciales y esenciales sobrepasan los requerimientos mínimos establecidos por los organismos internaciones en los tres alimentos.

Palabras clave: alimento, cachorro, macronutriente, aminoácidos, proteína, grasa, fibra

\section{Abstract}

The aim of this study was to determine the nutritional quality of three «Premium» commercial foods for puppies manufactured and marketed in Ecuador, as well as to determine the sensitivity of the near infrared spectroscopy (NIRS) technique for the assessment of the macronutrients of these foods. The nutritional characteristics of three brands of food were analysed, taking samples from four batches per brand. The macronutrients (protein, fat, fibre, starch, ash and moisture) were determined using the NIRS technique, a test that was duplicated in two laboratories with different equipment. In addition, the amino acid profile was determined by liquid chromatography (HPLC). In the proximal analysis, the three foods meet the minimum macronutrient requirements to guarantee the development and health of the puppies, although one of them showed better nutritional composition. The NIRS technique as a rapid test is stable to analyse the components of dry pet food; however, it presented variability when measuring mainly the fat concentration $(\mathrm{p}=0.029)$ in the three foods, although it also occurred in one for fibre and ash contents. The concentration of non-essential and essential amino acids exceeded the minimum requirements established by international organizations in the three foods.

Key words: food, puppy, macronutrient, amino acids, protein, fat, fibre

\section{INTRODUCCIÓN}

En el mercado de alimentos para animales de compañía se puede encontrar una gran cantidad de marcas comerciales a nivel mundial, algunas de ellas distribuidas internacionalmente. Estos alimentos son ofrecidos como suplementos o como alimentos balanceados completos, y son clasificados como económicos, premium o super premium en base a las materias primas con los que fueron elaborados, lo cual influye en su costo de producción y en su precio (Gomez, 2012).

El término de «alimento funcional», es un concepto aplicado a los alimentos balanceados, pues proporcionan beneficios para la salud con base a las características individuales de cada animal (edad, raza, condición fisiológica), no siendo solo utilizados como factores de saciedad o para proveer nutrientes esenciales, sino, además, favorecen el óptimo metabolismo incluso frente a la enfermedad (Chandler y Takashima, 2014). Se debe tener en cuenta que la correcta nutrición influye en la calidad de vida y en la longevidad de los animales (Laflamme, 2005). Es así que la nutrición adecuada en las etapas juveniles debe precautelarse para evitar deficiencias que pueden repercutir en el perro adulto o geriátrico (Debenecker et al., 2010).

Los neonatos (caninos de 1 hasta 30 días), al igual que otros mamíferos se alimentan del calostro o leche materna, la cual es nutricionalmente equilibrada para cada especie en particular (Mila et al., 2017). Usualmente, los cachorros caninos comienzan a comer alimentos semisólidos poco después 
de la erupción de los dientes deciduos, pero la leche sigue siendo suficiente para mantener el crecimiento y el desarrollo hasta las 45 semanas de edad, tiempo en el cual deben ser alimentados con concentrados de la fase de crecimiento (Hutter, 1991). En este sentido, Case (2012) indica un estado de transición nutricional entre las 3 a las 5 semanas de edad, donde se producen cambios fisiológicos en el tubo digestivo, a fin de adaptarse al nuevo alimento que recibirán luego del destete. En su etapa juvenil (entre 2 y 10 meses en perros de raza pequeña o hasta los 18 meses en razas grandes), el perro tiene un mayor requerimiento de proteínas, energía y calcio que un adulto (Dobenecker et al., 2010). La dieta también debe ser de alta calidad y fácil de digerir, sin sobrealimentar a los animales, principalmente con aportes exagerados de calcio, ya que esto puede predisponer a problemas esqueléticos en perros de razas grandes y a obesidad en los animales alimentados con dietas altas en energía y bajas en proteína (Lauten, 2006).

Las empresas dedicadas a la producción de alimentos para perros cuentan con centros de investigación para evaluar la calidad nutricional de sus alimentos. Asimismo, existen entes reguladores como la National Research Council (NRC), the Association of American Feed Control Officials (AAFCO) en América o la Federación Europea de Fabricantes de Alimentos para Animales de Compañía (FEDIAF) quienes establecen los requerimientos nutricionales que sirven de base para la formulación de las dietas y en ciertas regiones controlan la calidad de los alimentos comercializados (Leiva et al., 2019). En América Latina en general, cada país tiene las entidades gubernamentales para emisión de registros de calidad; sin embargo, el control y la evaluación de los alimentos comercializados no es una constante, siendo que las empresas que elaboran los alimentos balanceados para animales de compañía utilizan los parámetros estandarizados por las unidades americanas o europeas como guía de formulación (Gomez, 2012).
En el Ecuador se ha evidenciado cachorros con deficiencias nutricionales clínicas, así como ciertas deficiencias en el desarrollo de cachorros alimentados con dietas comerciales económicas, lo cual puede estar asociado a variaciones en la composición de los alimentos para cachorros producidos y comercializados en el país. Actualmente, de acuerdo con la Agencia Ecuatoriana de Aseguramiento de la Calidad del Agro (AGROCALIDAD) existen seis marcas de alimentos para cachorros, siendo tres de ellas, según los médicos veterinarios de clínicas de varias ciudades, las más comercializadas en el país, por lo que este estudio evaluó la calidad nutricional de estos alimentos.

\section{MATERIALES y Métodos}

\section{Muestras}

El estudio incluyó alimentos Premium para cachorros de tres marcas comerciales de fabricación nacional que son las más comercializadas en la ciudad de Quito y en general en el país (Guerra, 2020), lo cual fue corroborado mediante comunicaciones personales de médicos veterinarios y personal de ventas en consultorios, clínicas veterinarias, tiendas de mascotas, etc. Se tomaron muestras de los alimentos en diferentes puntos de venta, tomando en cuenta la marca y el número de lote de producción del alimento.

Se trabajó con cuatro lotes (repeticiones) de cada marca (A1, A2, A3, n=12 muestras). Cada muestra fue de $2 \mathrm{~kg}$, las cuales fueron divididas en tres submuestras de 550 g y remitidas a tres laboratorios para los análisis correspondientes.

\section{Calidad Nutricional}

Se determinó la composición nutricional de los alimentos balanceados mediante un análisis proximal a fin de determinar los niveles de humedad, proteína, grasa, fibra, almi- 
dón y cenizas. Se utilizó la técnica de espectroscopía por infrarrojo cercano (NIRS). Asimismo, para verificar posibles variaciones de los resultados se utilizaron dos equipos NIRS, uno PERTEN, modelo NIRS DA 7200, en la empresa Bioalimentar (BA) y el otro FOSS, modelo NIRS DS 2500, en la empresa Trow Nutrition (TN).

En forma paralela, se determinó la materia seca (MS) con un analizador termogravimétrico LECO TGA 701, proteína cruda (PC) por el método de Dumas con un equipo vario MAX cube (Elementar) y aminoácidos con un equipo HPLC Biochrom 30/30+ (Biochrom) en los laboratorios Evonic Industries AG.

\section{Análisis Estadístico}

Se realizaron estadísticas descriptivas sobre los datos obtenidos en el análisis proximal y en el aminograma. En el mismo conjunto de datos se realizó la prueba de normalidad Shapiro Wilk y un análisis de varianza para verificar las variaciones entre laboratorios (análisis proximal) y entre alimentos. La prueba de Tukey se utilizó para determinar diferencias significativas entre alimentos. Asimismo, se verificó la normalidad de los datos con la prueba de Kruskall Wallis y se aplicó la prueba de Wilcoxon (no paramétricos) para posicionar los alimentos. Se utilizó un valor de $\alpha=0.05$ como nivel de significancia para todas las pruebas de hipótesis. Los datos fueron analizados empleando el paquete estadístico R Studio.

\section{Resultados}

En el Cuadro 1 se presentan los resultados generales de la composición nutricional de los alimentos comerciales. Si bien se observa que de manera general los valores se encuentran dentro de los requerimientos establecidos en la literatura para perros, en el Cuadro 2 se puede notar diferencias entre marcas comerciales. Así, el alimento A3 pre- sentó un mayor nivel de nivel de proteína, grasa y cenizas que los alimentos AI y A2 $(p<0.05)$, no habiendo diferencias significativas entre ellos. Contrariamente, el porcentaje de fibra fue superior en el alimento A1 en comparación con los alimentos A2 y A3 $(\mathrm{p}<0.05)$. Asimismo, el porcentaje de almidón fue significativamente diferente en los tres alimentos, siendo mayor en A2 y menor en A3 ( $p<0.05)$. Finalmente, el alimento A1 fue significativamente más seco que los alimentos A2 y A3 $(p<0.05)$

Al comparar los resultados de composición nutricional de los alimentos mediante la técnica NIRS en dos laboratorios se encontraron diversas diferencias, indicando la posible variabilidad de resultados que pueden obtenerse con esta técnica entre laboratorios de análisis (Cuadro 3). Se observó diferencias significativas en los porcentajes de grasa para los alimentos A1 y A2, para la fibra en el alimento A1 y para cenizas en el alimento A3 ( $<<0.05)$. Por otro lado, no hubo mayores diferencias para los porcentajes de humedad, proteína y almidón.

Se contrastaron los niveles nutricionales de los alimentos obtenidos en el análisis de laboratorio con los niveles citados por cada fabricante en el empaque comercial correspondiente (Cuadro 4). Los niveles de proteína fueron menores en los alimentos A1 y 2 en comparación a lo indicado en las etiquetas de los productos. En forma similar el contenido de fibra fue menor en los alimentos $\mathrm{A} 2 \mathrm{y}$ $\mathrm{A} 3$, y el contenido de grasa fue superior en los alimentos A2 y A3 y de cenizas en los tres alimentos $(\mathrm{p}<0.05)$.

La calidad de la proteína fue analizada mediante la determinación del perfil de aminoácidos. Los perfiles fueron comparados entre los tres alimentos, así como con los mínimos referenciales presentados por las asociaciones que regulan estos requerimientos mínimos (AAFCO, 2016; FEDIAF, 2017). Los resultados se muestran en el Cuadro 5. 
Cuadro 1. Composición nutricional de alimentos balanceados para perros de tres marcas comercializadas en Ecuador

\begin{tabular}{lcccc}
\hline Nutriente & Media & Mediana & Mínimo & Máximo \\
\hline Proteína (\%) & 26.58 & 25.5 & 22.2 & 32.2 \\
Grasa (\%) & 12.26 & 11.39 & 9.21 & 16.7 \\
Humedad (\%) & 7.41 & 7.23 & 5.5 & 9.54 \\
Fibra (\%) & 2.73 & 2.73 & 1.7 & 4.0 \\
Almidón (\%) & 32.13 & 31.88 & 24.3 & 43.5 \\
Cenizas (\%) & 6.75 & 6.81 & 4.7 & 9.9 \\
\hline
\end{tabular}

Cuadro 2. Variación en el contenido de macronutrientes de alimentos balanceados para perros de tres marcas comercializadas en Ecuador

\begin{tabular}{|c|c|c|c|c|c|c|c|}
\hline Nutriente & A1 & A2 & A3 & Media & $\begin{array}{l}\text { CV } \\
(\%)\end{array}$ & Kruskal & $p$ value \\
\hline $\begin{array}{l}\text { Proteína } \\
(\%)\end{array}$ & $24.26 \pm 1.16^{\mathrm{a}}$ & $24.51 \pm 1.16^{\mathrm{a}}$ & $30.98 \pm 0.66^{\mathrm{b}}$ & 26.58 & 12.52 & 15.39 & 0.00045 \\
\hline $\begin{array}{l}\text { Grasa } \\
(\%)\end{array}$ & $10.96 \pm 1.06^{\mathrm{a}}$ & $10.24 \pm 0.94^{\mathrm{a}}$ & $15.58 \pm 0.56^{\mathrm{b}}$ & 12.25 & 20.9 & 16.34 & 0.00028 \\
\hline $\begin{array}{l}\text { Humedad } \\
(\%)\end{array}$ & $6.21 \pm 0.43^{\mathrm{a}}$ & $8.20 \pm 0.82^{\mathrm{b}}$ & $7.85 \pm 0.84^{b}$ & 7.41 & 15.12 & -- & 0.00004 \\
\hline Fibra (\%) & $3.26 \pm 0.65^{\mathrm{a}}$ & $2.39 \pm 0.44^{b}$ & $2.54 \pm 0.34^{b}$ & 2.73 & 22.5 & -- & 0.00496 \\
\hline $\begin{array}{l}\text { Almidón } \\
(\%)\end{array}$ & $32.3 \pm 1.58^{\mathrm{a}}$ & $37.73 \pm 2.36^{\mathrm{b}}$ & $26.37 \pm 1.13^{\mathrm{c}}$ & 32.13 & 15.93 & -- & 0.0000 \\
\hline $\begin{array}{l}\text { Cenizas } \\
(\%)\end{array}$ & $6.83 \pm 0.68^{\mathrm{a}}$ & $6.05 \pm 0.83^{\mathrm{a}}$ & $7.4 \pm 1.16^{\mathrm{b}}$ & 6.75 & 15.4 & -- & 0.0256 \\
\hline
\end{tabular}

A1, A2, A3: Alimentos de las tres marcas comerciales analizadas (cuatro repeticiones-lotes - por marca)

En general, los valores encontrados superan los requerimientos mínimos establecidos; sin embargo, se puede apreciar que, con excepción de la glicina y la arginina, los niveles de aminoácidos no esenciales y esenciales, respectivamente, difieren significativamente entre alimentos $(\mathrm{p}<0.05)$.

\section{Discusión}

En el presente estudio se pudo determinar que los niveles de los macronutrientes (proteína, grasa, fibra, almidón y ceniza) en los alimentos Premium para cachorros fabri- 
Cuadro 3. Variación de componentes nutricionales de alimentos balanceados para perros de tres marcas comercializadas en Ecuador determinados por la técnica de espectroscopia por infrarrojo cercano (NIRS) según el laboratorio de análisis

\begin{tabular}{lcccccc}
\hline \multirow{2}{*}{ Nutriente } & \multicolumn{2}{c}{ Alimento 1 } & \multicolumn{2}{c}{ Alimento 2 } & \multicolumn{2}{c}{ Alimento 3 } \\
\cline { 2 - 7 } & A1BA $^{1}$ & A1TN $^{2}$ & A2BA & A2TN & A3BA & A3TN \\
\hline $\begin{array}{l}\text { Proteína } \\
(\%)\end{array}$ & $24.89 \pm 0.92^{\mathrm{a}}$ & $23.63 \pm 1.20^{\mathrm{a}}$ & $24.55 \pm 1.45^{\mathrm{a}}$ & $24.48 \pm 1.03^{\mathrm{a}}$ & $30.61 \pm 0.40^{\mathrm{a}}$ & $31.35 \pm 0.70^{\mathrm{a}}$ \\
$\begin{array}{l}\text { Grasa } \\
(\%)\end{array}$ & $11.74 \pm 0.87^{\mathrm{a}}$ & $10.18 \pm 0.49^{\mathrm{b}}$ & $9.42 \pm 0.19^{\mathrm{a}}$ & $11.05 \pm 0.52^{\mathrm{b}}$ & $15.29 \pm 0.33^{\mathrm{a}}$ & $15.88 \pm 0.64^{\mathrm{a}}$ \\
$\begin{array}{l}\text { Humedad } \\
\text { \%) }\end{array}$ & $6.25 \pm 0.48^{\mathrm{a}}$ & $6.18 \pm 0.46^{\mathrm{a}}$ & $8.69 \pm 0.70^{\mathrm{a}}$ & $7.70 \pm 0.67^{\mathrm{a}}$ & $8.07 \pm 0.82^{\mathrm{a}}$ & $7.63 \pm 0.92^{\mathrm{a}}$ \\
$\begin{array}{l}\text { Fibra } \\
(\%)\end{array}$ & $2.70 \pm 0.25^{\mathrm{a}}$ & $3.83 \pm 0.29^{\mathrm{b}}$ & $2.56 \pm 0.51^{\mathrm{a}}$ & $2.23 \pm 0.36^{\mathrm{a}}$ & $2.86 \pm 0.10^{\mathrm{a}}$ & $2.23 \pm 0.10^{\mathrm{a}}$ \\
$\begin{array}{l}\text { Almidón } \\
(\%)\end{array}$ & $32.22 \pm 1.49^{\mathrm{a}}$ & $32.38 \pm 1.68^{\mathrm{a}}$ & $36.12 \pm 1.62^{\mathrm{a}}$ & $39.35 \pm 3.11^{\mathrm{a}}$ & $25.61 \pm 0.87^{\mathrm{a}}$ & $27.13 \pm 1.39^{\mathrm{a}}$ \\
$\begin{array}{l}\text { Cenizas } \\
(\%)\end{array}$ & $6.27 \pm 0.38^{\mathrm{a}}$ & $7.40 \pm 0.29^{\mathrm{a}}$ & $6.70 \pm 0.26^{\mathrm{a}}$ & $5.4 \pm 0.66^{\mathrm{a}}$ & $6.62 \pm 0.38^{\mathrm{a}}$ & $8.18 \pm 1.18^{\mathrm{b}}$ \\
\hline
\end{tabular}

${ }^{1}$ BA: Laboratorio Bioalimentar; ${ }^{2}$ TN: Laboratorio Trow Nutrition

a,b Superíndices diferentes dentro de filas para cada alimento indican diferencias significativas entre laboratorios $(p<0.05)$

Cuadro 4. Valores nutricionales determinados en el análisis proximal y los citados por los fabricantes en las etiquetas de los empaques de alimentos balanceados para perros de tres marcas comercializadas en Ecuador

\begin{tabular}{lcccccc}
\hline \multicolumn{1}{c}{ Nutriente } & A1 & EMPA1 & A2 & EMPA2 & A3 & EMPA3 \\
\hline Proteína (\%) & $24.26 \pm 1.16^{\mathrm{a}}$ & $27^{\mathrm{b}}$ & $24.51 \pm 1.16^{\mathrm{a}}$ & $27^{\mathrm{b}}$ & $30.98 \pm 0.66^{\mathrm{a}}$ & $30^{\mathrm{a}}$ \\
Grasa (\%) & $10.96 \pm 1.06^{\mathrm{a}}$ & $10^{\mathrm{a}}$ & $10.24 \pm 0.94^{\mathrm{a}}$ & $8^{\mathrm{b}}$ & $15.58 \pm 0.56^{\mathrm{a}}$ & $15^{\mathrm{b}}$ \\
Humedad (\%) & $6.21 \pm 0.43^{\mathrm{a}}$ & $12^{\mathrm{b}}$ & $8.20 \pm 0.82^{\mathrm{a}}$ & $12^{\mathrm{b}}$ & $7.85 \pm 0.84^{\mathrm{a}}$ & $12^{\mathrm{b}}$ \\
Fibra (\%) & $3.26 \pm 0.65^{\mathrm{a}}$ & $3^{\mathrm{a}}$ & $2.39 \pm 0.44^{\mathrm{a}}$ & $3.5^{\mathrm{b}}$ & $2.54 \pm 0.34^{\mathrm{a}}$ & $5^{\mathrm{b}}$ \\
Cenizas (\%) & $6.83 \pm 0.68^{\mathrm{a}}$ & $8^{\mathrm{b}}$ & $6.05 \pm 0.83^{\mathrm{a}}$ & $8^{\mathrm{b}}$ & $7.4 \pm 1.16^{\mathrm{a}}$ & $10^{\mathrm{b}}$ \\
\hline
\end{tabular}

A1, A2, A3: Alimentos de las tres marcas comerciales analizadas (cuatro repeticiones - lotes - por marca) EMPA1, EMPA2, EMPA3: Etiquetas de los tres alimentos comerciales

$a, b$ Superíndices diferentes dentro de filas para cada alimento indican diferencias significativas $(p<0.05)$

cados en el Ecuador cumplen con los requerimientos mínimos establecidos por las asociaciones internacionales como la AAFCO (2016) y la FEDIAF (2017); sin embargo, se pudo apreciar que ninguna de ellas cumple a cabalidad con los niveles registrados en sus etiquetas. Esta diferencia, de acuerdo con lo establecido por la Agencia Ecuatoriana de Aseguramiento de la Calidad del Agro, en su Resolución 0018 , artículo 1, y por la norma INEN 187, puede llevar a confusión en los consumidores (propietarios de mascotas que 
Cuadro 5. Perfil de aminoácidos de alimentos balanceados para perros de tres marcas comercializadas en Ecuador

\begin{tabular}{|c|c|c|c|c|c|}
\hline \multirow{2}{*}{ Nutriente } & $\mathrm{x} . \pm \mathrm{DE}$ & $\mathrm{x} . \pm \mathrm{DE}$ & $\mathrm{x} . \pm \mathrm{DE}$ & \multirow{2}{*}{$\mathrm{x} . \pm \mathrm{DE}$} & \multirow{2}{*}{$\mathrm{CV} \%$} \\
\hline & A1 & $\mathrm{A} 2$ & A3 & & \\
\hline $\begin{array}{l}\text { Materia seca } \\
\mathrm{g} / 100 \mathrm{~g}\end{array}$ & $93.89 \pm 0.63$ & $92.20 \pm 0.68$ & $92.40 \pm 0.74$ & $92.83 \pm 1$ & 1.08 \\
\hline Proteína. g/100 g & $27.31 \pm 0.76$ & $27.97 \pm 1.73$ & $32.43 \pm 1.06$ & $29.24 \pm 2.63$ & 8.99 \\
\hline \multicolumn{6}{|c|}{ Aminoácidos no esenciales. g/100 g } \\
\hline Alanina & $1.62 \pm 0.07^{\mathrm{a}}$ & $1.85 \pm 0.15^{\mathrm{b}}$ & $2.41 \pm 0.10^{\mathrm{c}}$ & $1.96 \pm$ & 18.38 \\
\hline Ac. aspártico & $2.20 \pm 0.07 \mathrm{a}$ & $2.18 \pm 0.08^{\mathrm{ab}}$ & $2.33 \pm 0.06^{\mathrm{ab}}$ & $2.24 \pm 0.09$ & 4.42 \\
\hline Ac. glutámico & $4.32 \pm 0.06^{\mathrm{a}}$ & $4.15 \pm 0.29^{\mathrm{a}}$ & $5.52 \pm 0.27^{\mathrm{b}}$ & $4.66 \pm 0.67$ & 14.39 \\
\hline Glicina & $2.08 \pm 0.14$ & $2.01 \pm 0.12$ & $2.18 \pm 0.05$ & $2.09 \pm 0.12$ & 5.97 \\
\hline Serina & $1.13 \pm 0.04^{\mathrm{a}}$ & $1.16 \pm 0.08^{\mathrm{a}}$ & $1.44 \pm 0.04^{\mathrm{b}}$ & $1.24 \pm 0.15$ & 12.51 \\
\hline \multicolumn{6}{|c|}{ Aminoácidos esenciales. $\mathrm{g} / 100 \mathrm{~g}$} \\
\hline Arginina & $1.78 \pm 0.08$ & $1.67 \pm 0.07$ & $1.70 \pm 0.03$ & $1.72 \pm 0.07$ & 4.53 \\
\hline Histidina & $0.60 \pm 0.005^{\mathrm{a}}$ & $0.59 \pm 0.02^{\mathrm{a}}$ & $0.66 \pm 0.02^{b}$ & $0.62 \pm 0.03$ & 5.97 \\
\hline Isoleucina & $1.07 \pm 0.05^{\mathrm{a}}$ & $1.10 \pm 0.05^{\mathrm{a}}$ & $1.25 \pm 0.05^{\mathrm{b}}$ & $1.14 \pm 0.09$ & 7.94 \\
\hline Leucina & $1.96 \pm 0.05^{\mathrm{a}}$ & $2.31 \pm 0.21^{\mathrm{b}}$ & $3.41 \pm 0.19^{c}$ & $2.56 \pm 0.66$ & 25.85 \\
\hline Lisina & $1.46 \pm 0.05^{\mathrm{a}}$ & $1.47 \pm 0.06^{\mathrm{a}}$ & $1.24 \pm 0.02^{\mathrm{b}}$ & $1.39 \pm 0.11$ & 8.45 \\
\hline Cisteína & $0.39 \pm 0.02^{\mathrm{a}}$ & $0.38 \pm 0.03^{\mathrm{a}}$ & $0.48 \pm 0.01^{\mathrm{b}}$ & $0.42 \pm 0.051$ & 12.06 \\
\hline Metionina & $0.48 \pm 0.03^{\mathrm{a}}$ & $0.57 \pm 0.03^{b}$ & $0.69 \pm 0.04^{\mathrm{c}}$ & $0.59 \pm 0.09$ & 15.99 \\
\hline Met-Cis & $0.88 \pm 0.02^{\mathrm{a}}$ & $0.96 \pm 0.07^{\mathrm{a}}$ & $1.18 \pm 0.06^{\mathrm{b}}$ & $1.01 \pm 0.13$ & 13.75 \\
\hline Fenilalanina & $1.12 \pm 0.02^{\mathrm{a}}$ & $1.18 \pm 0.08^{\mathrm{a}}$ & $1.55 \pm 0.08^{\mathrm{b}}$ & $1.28 \pm 0.20$ & 16.1 \\
\hline Treonina & $0.98 \pm 0.04^{\mathrm{a}}$ & $1.00 \pm 0.05^{\mathrm{a}}$ & $1.11 \pm 0.03^{b}$ & $1.03 \pm 0.07$ & 6.96 \\
\hline Valina & $1.31 \pm 0.05^{\mathrm{a}}$ & $1.35 \pm 0.07^{\mathrm{a}}$ & $1.52 \pm 0.06^{\mathrm{b}}$ & $1.4 \pm 0.11$ & 8 \\
\hline Prolina & $1.83 \pm 0.11^{\mathrm{a}}$ & $1.75 \pm 0.10^{\mathrm{a}}$ & $2.44 \pm 0.10^{\mathrm{b}}$ & $2.01 \pm 0.35$ & 17.54 \\
\hline Triptófano & $0.27 \pm 0.003^{\mathrm{a}}$ & $0.25 \pm 0.01^{\mathrm{b}}$ & $0.25 \pm 0.004^{\mathrm{b}}$ & $0.26 \pm 0.01$ & 4.18 \\
\hline \multicolumn{6}{|c|}{ Otros } \\
\hline Amoniaco & $0.56 \pm 0.002^{\mathrm{a}}$ & $0.57 \pm 0.03^{\mathrm{a}}$ & $0.74 \pm 0.04^{\mathrm{b}}$ & $0.62 \pm 0.09$ & 14.56 \\
\hline $\mathrm{AA}+\mathrm{Amoniaco}$ & $25.26 \pm 0.89^{\mathrm{a}}$ & $25.62 \pm 1.63^{\mathrm{a}}$ & $30.99 \pm 1.16^{\mathrm{b}}$ & $27.3 \pm 2.97$ & 10.88 \\
\hline Aasin Amoniaco & $24.70 \pm 0.89^{\mathrm{a}}$ & $25.05 \pm 1.6^{\mathrm{a}}$ & $30.25 \pm 1.12^{\mathrm{b}}$ & $26.67 \pm 2.88$ & 10.8 \\
\hline
\end{tabular}

A1, A2, A3: Alimentos de las tres marcas comerciales analizadas (cuatro repeticiones - lotes - por marca) $a, b$ Superíndices diferentes dentro de filas indican diferencias significativas $(p<0.05)$

toman en cuenta los parámetros establecidos en la etiqueta). Si bien los niveles de proteína están acordes a lo recomendado por varios autores (Case, 2012; Daumas et al., 2014), los niveles de proteína en los alimentos A1 y A 2 presentan $3 \%$ menos que el A3, pudiendo llegar a afectar el desarrollo de los cachorros, especialmente por el tipo de proteína empleada en la preparación de los alimentos (Venturini et al., 2018). Por otro lado, la medición mediante la técnica NIRS no presentó diferencias entre laboratorios. 
La técnica NIRS es una técnica confiable para la valoración rápida de los alimentos secos para mascotas (Hervera et al., 2009); sin embargo, se debe tener en cuenta que se requiere realizar controles y calibraciones continuas de los equipos y las curvas. En el presente estudio se encontraron diferencias entre laboratorios para la determinación de grasa, lo cual concuerda con otros reportes que indican que el NIRS no es estable para grasa (Alomar et al., 2006; De Marchi et al., 2016).

En el alimento A1 se determinó un menor contenido de fibra por parte del laboratorio BA $(2.70 \%)$ y en comparación con el laboratorio TN $(3.83 \%)(p=0.00066)$, siendo este un factor que de no ser valorado de manera adecuada puede llevar a los fabricantes de alimentos balanceados a incluir niveles elevados de fibra en el alimento seco. Si bien niveles de fibra altos se han empleado para corregir cuadros asociados a la obesidad en perros adultos (Wambacq et al., 2016), en los cachorros el exceso de fibra en la dieta puede ocasionar aumento del peristaltismo intestinal y cuadros diarreicos. Esta variación asociada a laboratorio solo se observó para el alimento A1, de modo que se puede descartar el método de análisis. La FAO, por otro lado, recomienda los métodos gravimétricos (sencillos y rápidos) y enzimático químicos (complejos y lentos), por ejemplo, HPLC y cromatografía de gases (GLC) o colorimetría (FAO, 1997; de Godoy et al., 2013).

Los valores de almidón no presentaron diferencia significativa entre laboratorios, pero se aprecia una diferencia importante $\left(\mathrm{p}=1.28 \mathrm{E}^{-09}\right)$ entre alimentos $(32.3,37.73 \mathrm{y}$ $26.37 \%$ para A1, A2 y A3, respectivamente). Los niveles de inclusión del almidón son manipulados por los fabricantes de alimentos balanceados extruidos para expandir las croquetas a fin de obtener una textura más apropiada (Goi et al., 2020). No obstante, el almidón en exceso puede provocar intolerancia alimentaria, produciendo atrofia de las vellosidades intestinales, lo que induce a presentación de diarrea causada por una mala absorción (Grandjean y Butterwick, 2009).

El valor de humedad indicado por los fabricantes era de 12\%; sin embargo, los valores obtenidos en el estudio fueron bastante menores $(p=0.007)$ y con diferencias significativas entre alimentos de las tres marcas. Esto indica claramente que son empacados con un menor porcentaje de humedad (Cuadro 4), asumiendo este factor para la prevención del desarrollo de hongos o bacterias que son más frecuentes de presentarse en alimentos con mayor humedad (Laflamme et al., 2014; Olivry y Mueller, 2019), y si a esto se le suma también la alta concentración de grasa encontrada en el alimento A3, la probabilidad de descomposición del alimento podría ser alta (Case, 2012).

Los resultados del análisis del perfil de aminoácidos indican que los tres alimentos Premium superan los requerimientos mínimos de aminoácidos establecidos por la AFFCO (2016), garantizando un perfil aminoacídico que fomenta la salud y el bienestar de los animales. Las escasas variaciones en las concentraciones de aminoácidos entre los alimentos pueden estar asociadas a las materias primas utilizadas o al proceso de fabricación (Tjernsbekk et al., 2016).

\section{Conclusión}

- Los tres alimentos comerciales «Premium» para cachorros cumplen con los requerimientos nutricionales mínimos establecidos por los organismos gubernamentales.

- La técnica de espectroscopía por infrarrojo cercano (NIRS) es una prueba rápida que permite determinar la composición nutricional del producto terminado; sin embargo, se debe tener en cuenta la variabilidad de este método para la determinación del contenido de grasa y de fibra. 
- Los niveles de humedad en los tres alimentos fueron bajos con respecto a lo enunciado por los fabricantes.

\section{Agradecimientos}

A la empresa Evonik Industries AG y Evonik Operations GmbH por su colaboración en el análisis del perfil de aminoácidos incluido en el presente trabajo. Asimismo, a la empresa Bioalimentar por su colaboración y apertura para realizar esta investigación.

\section{Literatura Citada}

1. Alomar D, Hodgkinson S, Abarzúa D, Fuchslocher R, Alvarado C, Rosales E. 2006. Nutritional evaluation of commercial dry dog foods by near infrared reflectance spectroscopy. J Anim Physiol An N 90: 223-229. doi: 10.1111/ j.1439-0396.2005.00585.x

2. [AAFCO] Association of American Feed Control Officials. 2016. 2016 AAFCO Midyear Meeting Committee Reports. [Internet]. Available in: https:// www.aafco.org/Portals/0/SiteContent/ Meetings/Midyear/2016/2016_Midyear_Committee_-Reports_w_cover.pdf.

3. Case L, Hayec M, Daristotle L, Foess M. 2012. Canine and feline nutrition. A resource for companion animal professionals. $3^{\text {rd }}$ ed. Inter Médica. 562 p.

4. Chandler ML, Takashima G. 2014. Nutritional concepts for the veterinary practitioner. Vet Clin N Am-Small 44: 645-666. doi: 10.1016/j.cvsm.2014.03.009

5. Daumas $C$, Paragon BM, Thorin $C$, Martin L, Dumon H, Ninet S, Nguyen P. 2014. Evaluation of eight commercial dog diets. J Nutr Sci 3: e63. doi: 10.1017/ jns. 2014.65

6. de Godoy MR, Kerr KR, Fahey GC. 2013. Alternative dietary fiber sources in companion animal nutrition. Nutrients 5: 3099-3117. doi: 10.3390/nu5083099
7. De Marchi M, Righi F, Meneghesso M, Manfrin D, Ricci R. 2018. Prediction of chemical composition and peroxide value in unground pet foods by near-infrared spectroscopy. J Anim Physiol An N 102: 337-342. doi: 10.1111/ jpn.12663

8. Dobenecker B, Frank V, Kienzle E. 2010. High calcium intake differentially inhibits nutrient and energy digestibility in two different breeds of growing dogs. J Anim Physiol An N 94: e109-14. doi: 10.1111/j.1439-0396.2010.00989.x

9. [FAO] Organización de las Naciones Unidas para la Alimentación y la Agricultura. 1997. Análisis de fibra dietética. En: Producción y manejo de datos de composición química de alimentos en nutrición. [Internet]. Disponible en: http://www.fao.org/3/ah833s/ah833s18.htm

10. [FEDIAF] Federación Europea de Fabricantes de Alimentos para Animales de Compañía. 2017. Guías nutricionales para alimentos completos y complementarios para perros y gatos. 102 p. [Internet]. Disponible en: https:// www.um.es/documents/14554/744854/ Guias-Nutricionales-FEDIAF-es2017.pdf/410142b0-9ad7-4752-a0a73b102b1dc3c0

11. Goi A, Simoni M, Righi F, Visentin G, De Marchi M. 2020. Application of a handheld near-infrared spectrometer to predict gelatinized starch, fiber fractions, and mineral content of ground and intact extruded dry dog food. Animals 10: 1660. doi: 10.3390/ani10091660

12. Gomez L. 2012. Introducción a la nutrición de caninos y felinos. J Agric Anim Sci 1: 52-61. doi: 10.1590/1518-8345.0979.2734

13. Grandjean D, Butterwick R. 2009. Libro de bolsillo WALTHAM ${ }^{\circledR}$ sobre nutrición esencial de gatos y perros nutrición esencial de gatos y perros. Waltham. $64 \mathrm{p}$. 
14. Guerra E. 2020. Proyecto de factibilidad para la creación de una comercializadora de alimento y accesorios para mascotas en la ciudad de Quito. Tesis de Maestría. Quito, Ecuador: Univ. Politécnica Salesiana. $74 \mathrm{p}$.

15. Hervera M, Baucells MD, González G, Pérez E, Castrillo C. 2009. Prediction of digestible protein content of dry extruded dog foods: comparison of methods. J Anim Physiol An 93: 366-372. doi: 10.1111/j.1439-0396.2008.00870.x

16. Hutter ER. 1991. Nutrición en caninos y felinos. Edición digital [Internet]. Disponible en: https://studylib.es/doc/ 7483443/nutrici\%C3\%B3n-en-caninos$\mathrm{y}$-felinos.

17. Laflamme D, Izquierdo O, Eirmann L, Binder S. 2014. Myths and misperceptions about ingredients used in commercial pet foods. Vet Clin N AmSmall 44: 689-698. doi: 10.1016/ j.cvsm.2014.03.002

18. Laflamme DP. 2005. Nutrition for aging cats and dogs and the importance of body condition. Vet Clin NAm-Small 35: 713742. doi: 10.1016/j.cvsm.2004.12.011

19. Lauten SD. 2006. Nutritional risks to large-breed dogs: from weaning to the geriatric years. Vet Clin N Am-Small 36: 1345-1359. doi: 10.1016/j.cvsm.2006.09.003

20. Leiva A, Molina A, Redondo-Solano M, Artavia G, Rojas-Bogantes L, Granados-Chinchilla F. 2019. Pet food quality assurance and safety and quality assurance survey within the Costa Rican pet food industry. Animals 9: 980. doi: 10.3390/ani9110980

21. Mila H, Grellet A, Delebarre M, Mariani C, Feugier A, ChastantMaillard S. 2017. Monitoring of the newborn dog and prediction of neonatal mortality. Prev Vet Med 143: 11-20. doi: 10.1016/j.prevetmed.2017.05.005

22. Olivry T, Mueller RS. 2019. Critically appraised topic on adverse food reactions of companion animals (8): storage mites in commercial pet foods. BMC Vet Res 15: 385. doi: 10.1186/s12917-019-2102-7

23. Tjernsbekk MT, Tauson AH, Matthiesen CF, Ahlstrom O. 2016. Protein and amino acid bioavailability of extruded dog food with protein meals of different quality using growing mink () as a model. J Anim Sci 94: 3796-3804. doi: 10.2527/jas.2016-0526

24. Venturini KS, Sarcinelli MF, Baller MA, Putarov TC, Malheiros EB, Carciofi AC. 2018. Processing traits and digestibility of extruded dog foods with soy protein concentrate. J Anim Physiol An N 102: 1077-1087. doi: 10.1111/ jpn. 12894

25. Wambacq W, Rybachuk G, Jeusette I, Rochus K, Wuyts B, Fievez, V, Nguyen P, Hesta M. 2016. Fermentable soluble fibres spare amino acids in healthy dogs fed a low-protein diet. BMC Vet Res 12: 130. doi: 10.1186/s12917-016-0752-2 